\title{
"Publizistisches Apostolat" \\ Das Bild des (katholischen) Journalisten bei Johannes Paul II.
}

\author{
von Albert Steuer
}

„Die katholische Presse ist für die Verbreitung christlicher Grundsätze und die Verteidigung der Standpunkte der Katholiken ebenso wichtig wie zur Bildung einer gesunden und für jede gute Sache aufgeschlossenen öffentlichen Meinung. In einer Gesellschaft mit gehobenem Bildungsstand ist die Präsenz einer qualifizierten und nach verschiedenen Gattungen aufgefächerten katholischen Presse absolut notwendig. ... Diese Presse ist dazu bestimmt, die Gewissen zu erleuchten und falsche Interpretationen, Unterstellungen und Manipulationen zu zerstreuen - in Achtung vor anderen Meinungen und im vertrauensvollen Dialog. ... Sie ist dazu aufgerufen, im Leser jenen Urteilsprozeß in Gang zu setzen, der ihn zur befreienden und erlösenden Wahrheit hinführt." ${ }^{\text {Papst }}$ Johannes Paul II. bringt in diesen Worten auf eine - apologetisch akzentuierte Kurzformel, was das II. Vatikanische Konzil in seinem Dekret "Inter mirifica“ über die sozialen Kommunikationsmittel entfaltet und im Ersten Kapitel auf die knappe Forderung gebracht hat: „Die Kirche hat also ein ursprüngliches Recht darauf, jedes dieser sozialen Kommunikationsmittel zu benutzen und zu besitzen, soweit es für die christliche Erziehung und ihr Wirken zum Heile der Seele notwendig und nützlich ist." (IM 3)

In diesem kurzen Zitat umreißt Johannes Paul II., daß für ihn die spezifisch katholische Presse in erster Linie ein Instrument zur Evangelisierung bzw. 'Neuevangelisierung' ist, wie er es mit Blick auf Europa immer wieder fordert. Die Stimme der katholischen Presse gehört für ihn notwendig zur Medienwelt einer pluralistischen Gesellschaft, wo sie im Konzert der öffentlichen Meinung ihren Part zu übernehmen hat. Der oben angeführte kurze Auszug aus einer Ansprache des Papstes an italienische Bischöfe macht deutlich, was er von der Presse allgemein und von der katholischen Presse im Besonderen erwartet. Das Zitat umreißt zugleich auch das Paradigma, in dem Johannes Paul II. seine Erwartungen an die Medienschaffenden und insbesondere an die (katholischen) Journalisten entwickelt. Der folgende Beitrag will versuchen, das Bild, das sich Johannes Paul II. von den Journalisten - insbesondere von den katholischen Journalisten - macht, anhand seiner Ansprachen zu Medienthemen und seiner jährlichen Botschaften zum Welttag der sozialen Kommunikationsmittel in Umrissen und in den wichtigsten Grundzügen nachzuzeichnen. ${ }^{2}$

\section{Johannes Paul II. - ein „Medienpapst“?}

Die Einstellung der katholischen Kirche und des römischen Lehramtes gegenüber der Meinungs- und Pressefreiheit hat - insbesondere in den letzten Jahrzehnten unseres Jahrhunderts - einen tiefgreifenden Wandel erfahren.

Albert Steuer, Diplomtheologe, arbeitet seit 1984 als Journalist und Redakteur der Nachrichtenagentur KNA in Bonn. 
Während die Päpste des 19. und des beginnenden 20. Jahrhunderts von Gregor XVI. (1830-1846) bis Pius XI. (1922-1939) die freie Meinungsäußerung noch kategorisch und kämpferisch verurteilten, begann mit Pius XII. (1939-1958) eine Liberalisierung der kirchlichen Lehre durch die funktionale Neuinterpretation des Begriffs 'Öffentliche Meinung'.

Die Presse im modernen Sinn ist mit den großen Revolutionen des 19. Jahrhunderts entstanden und verstand bzw. versteht sich von daher zum einen als 'Kampfmittel' gegen die vorrevolutionären Herrschaftssysteme und zum anderen zunehmend als wichtiger und unentbehrlicher Faktor der demokratischen und pluralistischen Gesellschaften. Die Kirche hat früh auf die publizistischen Mittel zurückgegriffen und verhältnismäßig schnell auch eine eigene 'katholische; Presse entwickelt. Der Blick in die Geschichte zeigt aber, daß der Kirche die Nutzung der Presse oft durch außerkirchliche Entwicklungen aufgezwungen wurde; vielleicht liegt darin einer der tiefen Gründe für das bis in die Gegenwart doch ambivalente Verhältnis zwischen Kirche und Medien. Die im revolutionären Kontext entstandene Presse richtete ihre Angriffe nicht nur gegen die weltlichen Herrschaftssysteme, sondern auch gegen die Kirche, die bis dahin weithin eine enge Symbiose mit der säkularen Herrschaft eingegangen war.

In Gefolge von Revolution und Aufklärung ergaben sich geradezu naturgemäß Spannungen zwischen der Kirche als einer von Gott begründeten hierarchisch strukturierten Institution mit einem metaphysisch begründeten Dogmenund Moralkodex und den sich zu demokratischen und pluralistischen Systemen entwickelnden Gesellschaften sowie dem damit verbundenen Säkularisierungsprozeß.

Die Kirche als metaphysisch begründete Institution - und nach eigener Definition keine demokratische Gemeinschaft - entzieht sich den wie auch immer verfaßten demokratisch-pluralistischen Ordnungen säkularer Gesellschaften. Wenn auch die daraus resultierende „Grundspannung" zwischen Kirche als verfaßter Institution und säkularer Gesellschaft bis in die Gegenwart anhält und gerade auch unter dem gegenwärtigen Papst wieder stärker empfunden wird, so markiert doch Pius XII. den großen "Wendepunkt" in der kirchlichen Lehre zur Publizistik und Freiheit der Meinungsäußerung. Pius XII. betrachtete die zunehmende Demokratisierung der politischen Systeme der Neuzeit mit Sympathie und sah in der freien Meinungsäußerung und im Funktionieren der Öffentlichen Meinung eine wirksame und sinnvolle Garantie der Bürger- und Menschenrechte gegen eine das Individuum versklavende Gesellschaft. ${ }^{3}$ Öffentliche Meinung, wenn auch mit der Einschränkung, daß diese nur für jene Dinge gelten könne, „die der freien Diskussion überlassen sind“, ist für Pius XII. auch innerhalb der Kirche notwendig, weil diese „ein lebendiger Körper ist, und es würde etwas in ihrem Leben fehlen, wenn ihr die Öffentliche Meinung mangelte, ein Mangel, wofür die Schuld sowohl auf die Hirten wie die Gläubigen fiele". So ist es gleichermaßen auf die säkulare Gesellschaft wie auf die Gemeinschaft der Kirche zu beziehen, wenn Pius XII. die Öffentliche Meinung als „Mitgift jeder normalen Gesellschaft“ bezeichnet. ${ }^{4}$ Nach dieser hier nur andeutbaren Grundsteinlegung der „Wende" sind die Aussagen der Päpste Johannes XXIII. (1958-1963) und Johannes Paul I. (1978) insofern zu vernachlässi- 
gen, als ersterer voll durch das II. Vatikanische Konzil beansprucht wurde und letzterem nur eine 33tägige Amtszeit beschieden war. Weitere grundlegende kirchenamtliche Dokumente im Prozeß der Neubesinnung der Kirche in ihrem Verhältnis zum modernen medialen Kommunikationsgeschehen sind das Konzilsdekret "Inter mirifica“ über die sozialen Kommunikationsmittel Ü(04.12.1963) und vor allem die im Auftrag des Konzils erarbeitete Pastoralinstruktion "Communio et Progressio" (23.05.1971). Auf der Grundlage der Aussagen Pius XII. und den beiden genannten Dokumenten haben dann vor allem die Päpste Paul VI. (1963-1978) und Johannes Paul II. (seit 1978) eine in ihrer Grundstruktur doch sehr ähnliche „Ethik der Massenkommunikation“ entfaltet. ${ }^{5}$

Ohne die „Vorarbeit“ seiner unmittelbaren Vorgänger auf dem Stuhl Petri schmälern zu wollen: Johannes Paul II. versteht es wie kein Papst vor ihm, sich der Möglichkeiten der modernen Massenkommunikationsmittel zu bedienen. Es gelingt ihm immer wieder - "Naturtalent oder geschicktes Kalkül?" - , die Medien auf sich aufmerksam zu machen und für sein Pontifikat zu nutzen: „Auch ohne Worte versteht er es zu faszinieren durch seine Gesten, Handlungen und bildschirmgerechtes Agieren." Seine "neue offene Art der Mitteilung“ läßt ihn für viele Beobachter "gleichsam am Schnittpunkt jeder Kommunikation" stehen als einer, „der auf 'Medienpapst' gelernt zu haben scheint. "8 Eine unbestreitbare, allerdings wohl auch zweischneidige Begabung. Je länger sein Pontifikat dauert, desto deutlicher wird, daß M. Schmolke mit seiner kritischen Anmerkung so falsch nicht lag: „Ein Naturtalent im Umgang mit der Öffentlichkeit, Medienliebling und Medienopfer wie nie ein Papst vor ihm."

Unbestreitbar geht Johannes Paul II. in einer bisher so nicht erlebten Offenheit auf die Presse zu. Er sucht immer wieder die Chancen der Medien als „wunderbare und zeitgemäße Werkzeuge für die Verbreitung des Evangeliums ${ }^{“ 10}$, als Möglichkeit der Begegnung mit den Menschen und als ein Forum, um mit seiner Stimme für die "Stimmlosen" - die Unterdrückten, Verfolgten und Entrechteten - zu sprechen. Waren für Gregor XVI. noch jene vom "Irrsinn" geleitet, die die "verabscheuenswerte Freiheit der Presse" forderten ${ }^{11}$, so hat Johannes Paul II. gut hundert Jahre später keine Scheu, eben diese Pressefreiheit anzuerkennen und einzuklagen. Immer wieder sucht er bei seinen Auslandsreisen auch das Gespräch mit den Kultur- und Medienschaffenden und bezeichnet die Journalisten, in deren Tätigkeit er einen „bedeutenden Dienst an der Menschheit ${ }^{\text {"12 }}$ sieht, als „Freunde“. In einer neuen Art und Weise hat Johannes Paul II. die von "Inter mirifica" erhobene Forderung, daß es die Kirche als ihre Pflicht erachten müsse, „die Heilsbotschaft auch mit Hilfe der sozialen Kommunikationsmittel zu verkünden“13, und den in "Communio et Progressio" weiter präzisierten Wunsch nach Nutzung der Kommunikationsmittel für die Verkündigung ${ }^{14}$ zu einem grundlegenden Element seines Pontifikats gemacht.

\section{2. "Anthropozentrische“ Medienethik}

„Mit einem Wort: Bemüht Euch um die Förderung einer Kultur, die wahrhaftig dem Menschen entspricht in dem Bewußtsein, daß Ihr dadurch die Begegnung mit dem Glauben, den niemand zu fürchten hat, erleichtert." 15 Dieser Appell Johannes Paul II. streift alle wichtigen Elemente seiner "Lehre“ zur mo- 
dernen Massenkommunikation. Grundkriterium ist für den Papst der Mensch. Die Bedingungen und Möglichkeiten menschlicher Existenz in der Öffentlichkeit stellt er in seinen Medien-Ansprachen immer wieder in den Mittelpunkt. Der Mensch als konkretes Individuum ist ihm „zusammenfassendes Thema aller Wissenschaft und Kultur". Dieser Mensch, so leitet Johannes Paul II. aus seiner im Schöpfungsglauben verankerten Anthropologie $\mathrm{ab}^{16}$, ist auch „das Thema der Kirche“; ihm haben gerade auch die Kommunikationsmedien zu dienen, indem sie Gemeinschaftssinn stiften. Wie es Aufgabe der Kirche ist, so erwartet der Papst auch von den Medien, daß sie den Menschen als freie Person respektieren. Wissenschaft, Technologie, Kunst und Kultur, sind nach seiner Überzeugung für den Menschen Mittel, Welt und Leben zu deuten, seine epochale Situation auszuleuchten und die Höhen und Tiefen seiner Existenz zu erfassen. Dabei darf aber die Perspektive nicht nur auf das Diesseitig-Irdische gerichtet sein. Eine humane Anthropologie ist nur „authentisch“, wenn sie neben der irdisch-horizontalen auch der transzendent-vertikalen Dimension des Menschen Rechnung trägt. „Gebt dem Transzendenten Raum, das den Menschen mehr zum Menschen macht!" appelliert Johannes Paul II. immer wieder an Kultur- und Medienschaffende. ${ }^{17}$ Aus diesem anthropozentrischen Paradigma leitet er die übrigen Grundkategorien seiner Kommunikationslehre ab: die Verpflichtung gegenüber der Freiheit, den Kulturauftrag der Medien und ihre Brückenfunktion zwischen den Menschen, zwischen Gesellschaft und Kirche, zwischen Glauben und Kultur. Um diese Grundfragen kreisen immer wieder die in bemerkenswerter Quantität vorliegenden Botschaften, Ansprachen und Aussagen Johannes Paul II. zu Themen der modernen Massenkommunikation. Ein Motiv seines diesbezüglichen Engagements ist für den 'Papst aus dem Osten' wohl auch seine polnische Herkunft, sein Leben in einem Staatssystem, das bis vor kurzem aus ideologischen Gründen keine Pressefreiheit kannte. Gerade diese polnische Herkunft scheint aber auch ein Grund für einen 'Bruch' in den Aussagen Johannes Paul II. zum Thema Medien/katholische Medien zu sein: Zum einen hat er keine Schwierigkeit, vehement immer wieder die allgemeine Wahrung der Pressefreiheit anzumahnen und einzufordern. Zum anderen hat er aber von der spezifisch katholischen Presse doch ehe das Bild eines 'geschlossenen Systems', das affirmativ und apologetisch den christlichen Glauben und die Kirche nach 'außen' hin verteidigen soll. Dieses Verständnis wirkt sich auch auf die Erwartungen aus, die der erste Slawe auf dem Stuhl Petri an die katholischen Journalisten richtet. Als ein besonderes Unterscheidungsmerkmal der Medien-Ethik Johannes Paul II. gegenüber Paul VI. hat G. Deussen die intensive Ausrichtung auf den Menschen ausgemacht und von einer „anthropologischen Wende" gesprochen. ${ }^{18}$ Dem ist zwar grundsätzlich zuzustimmen, doch angesichts der Tatsache, daß Johannes Paul II. - wie auch seine Vorgänger - keine zusammenhängende Medien-Ethik entwirft, sondern sich meist aus aktuellen Anlässen und in Gelegenheitsansprachen zum Thema äußert, sollte man vielleicht vorsichtiger von der Vision einer anthropozentrisch orientierten Publizistik sprechen.

\section{Johannes Paul II. und die Journalisten}

Nachdem vorausgehend der Kontext umrissen wurde, der für das Verständnis der Erwartungen Johannes Paul II. an die "Medienschaffenden“ Vor- 
aussetzung ist, soll im folgenden das Bild, das sich der Papst von den (katholischen) Journalisten macht, nachgezeichnet und hinterfragt werden. Wie kein anderer Pontifex vor ihm hat Johannes Paul II. - vor allem durch seine zahlreichen Auslandsreisen, bei denen er sich von Journalisten begleiten läßt -, mit der Presse zu tun. So wie er Jesu Aussendungsbefehl an die Apostel - "geht hinaus zu allen Völkern" (Mt 28,19ff.) - mit einem neuen Sinn zu erfüllen sucht, so möchte er auch die Medien nutzen, das Evangelium „von den Dächern“ (Mt. $10,27)$ zu verkünden. Wenn er auch die Journalisten immer wieder als 'Freunde' anredet, so belegen seine Ansprachen an diesen Berufsstand und seine Auseinandersetzung mit dem Arbeitsfeld Presse, daß es ihm dabei nicht um billige Anbiederung geht. Vielmehr möchte er diese 'Freunde' mit konstruktiver Kritik begleiten und immer wieder zum Nachdenken über ihre Arbeit und die sog. Sachzwänge des journalistischen Alltags anstoßen. Auch darin liegt ein Motiv seines bemerkenswerten Medien-Engagements, denn er weiß um die 'Macht der Massenkommunikationsmittel', insbesondere der audiovisuellen Medien, zum Guten wie zum Schlechten. 'Wahrheit' und 'Freiheit' sind für Johannes Paul II. oberste Kriterien, nach denen sich der Journalist richten muß, damit die Medien - im Sinne von "Communio et Progressio“ - im Dienst der Gemeinschaft und des Fortschritts der menschlichen Gesellschaft stehen.

\section{1 „Diener der Wabrbeit" - Berufsbild der Journalisten}

„Eine besondere Verantwortung für die sozialen Kommunikationsmittel tragen die Journalisten ... und überhaupt alle, die irgendwie bei der Produktion und Verbreitung sozialer Kommunikationsmittel beteiligt sind. ... Ihre Information und Entwicklung können dem Menschen Segen oder Fluch bringen. Ihre Aufgabe wird es darum sein, die wirtschaftlichen, politischen und künstlerischen Belange so aufeinander abzustimmen, daß sie dem Gemeinwohl niemals zuwiderlaufen. ... Sie sollen auch dafür sorgen, daß Darbietungen religiösen Inhalts ernsten und erfahrenen Bearbeitern anvertraut und mit gebotener Ehrfurcht gestaltet werden“, heißt es im Konzilsdekret "Inter mirifica. ${ }^{19}$ Die hier angesprochene Ambivalenz der Macht und die Wirkung der Medien nimmt Johannes Paul II. immer wieder zum Anlaß, die Medienschaffenden zu Gewissenhaftigkeit, Wahrheitsliebe und Verantwortungsbewußtsein aufzurufen, mißt er ihnen doch „einen ungeheuren Einfluß auf die Öffentlichkeit, auf die Meinungsbildung und auf das Bewußtsein von Millionen" bei. ${ }^{20}$ Die Verpflichtung zur Wahrheit macht den Journalisten in den Augen des Papstes zum „Diener der Wahrheit" und zum „Zeugen“. Diese Mittler- und Dienstfunktion stellt Johannes Paul II. immer wieder heraus: „Seid vor allem Diener der Wahrheit, stellt Euch in den Dienst dessen, was aufbaut, was den Menschen bessert und ihm höhere Würde gibt. ${ }^{\text {"21 }}$ So setzt sich im Berufsideal, das der Papst grundsätzlich allen nicht nur den katholischen - Journalisten immer wieder vor Augen stellt, die Anthropozentrik und die Orientierung am Gemeinwohl fort. Aus dieser Perspektive entfaltet er - auch in Ansprachen vor säkularen Journalisten - ein geradezu missionarisches Verständnis der Pressearbeit, die er als „Berufung“, „Apostolat" und „Suche nach der Wahrheit" charakterisiert. ${ }^{22}$ Geradezu optimistisch erscheint seine Vision: „Wenn einmal die Geschichte des letzten Viertels dieses Jahrhunderts geschrieben wird, dann wird, dessen bin ich sicher, der große Beitrag der Medien für die Sache der Menschheit aufgezeichnet sein für künftige Generationen. " ${ }^{\text {3 } 3}$ 
Im Gegenzug zu diesem idealistischen Bild sieht Johannes Paul II. auf der anderen Seite, wenn auch die Erforschung der Medienwirkung innerhalb der Wissenschaft noch weithin am Anfang steht, ebenso den möglichen negativen Einfluß der Massenmedien, und zwar besonders auf sittlich-sexuellem Gebiet. Ein besonderes Anliegen ist ihm dabei der mögliche negative Einfluß auf die Kinder und Jugendlichen. Daher erwartet er von den Medienschaffenden eine verantwortungsbewußte Auswahl und ethische Orientierung. Einen als Objektivität verkappten Werte-Neutralismus lehnt er ab. Die Journalisten haben für Johannes Paul II. die verantwortungsgewichtige Möglichkeit, den „Pulsschlag unserer Zeit aufzunehmen und zu bestimmen" und wie ein Seismograph zu reagieren und agieren, denn von der Tätigkeit der Medien „hängt - wenigstens zum Teil - das moralische Verhalten so vieler Männer und Frauen ... ab." ${ }^{24}$ Von daher leitet Johannes Paul II. immer wieder auch den Appell an die Journalisten $\mathrm{ab}$, im Sinne einer umfassenden ganzheitlichen Anthropologie der transzendenten Dimension des Menschen Rechnung zu tragen und daher die Themen Religion und Kirche nicht auszuklammern. Diese Thematik verlangt allerdings dem Berichterstatter eine „noch größere Verantwortung“ ab: „Von daher ergibt sich die Notwendigkeit eines gesteigerten Einfühlungsvermögens, um sich voll und ganz in grundlegenden dogmatischen und moralischen Fragen mit der authentischen Stimme des Lehramtes zu identifizieren, d. h. einer Haltung dieser Liebe zur Kirche und der treuen Zusammenarbeit mit ihr. Nur so kann es eine konstruktive Arbeit geben, nur so wird vermieden, die christliche Botschaft zu zersetzen und die Gläubigen durch unannehmbare Einstellungen oder zerstörerische Kritik zu verwirren." ${ }^{25}$ Unter journalistischer Arbeit versteht Johannes Paul II. nicht nur gemäß einer allgemeinen Definition das „Sammeln und Verbreiten von Nachrichten durch öffentliche und rechtzeitige Berichterstattung", sondern darüber hinaus, in Achtung vor den Werten "das Gute gut und das Böse böse" zu nennen und "positiv zur Sensibilisierung und Erziehung der Empfänger im Sinn eines vollen menschlichen und christlichen Wachstums" beizutragen. ${ }^{26}$

Für Johannes Paul II. sind die Medien grundsätzlich Mittel zur Verwirklichung der Freiheit. Wie seine Vorgänger auf dem Stuhl Petri bestätigt und verteidigt er daher das Recht und die Pflicht zur Informationsfreiheit. Dabei geht der Papst primär von einem individualethischen Freiheitsbegriff aus, erweitert diesen aber um eine sozialethische Dimension, indem er vor den Manipulationsmöglichkeiten durch politische Macht, gesellschaftliche „pressure groups“ oder „Marktführern“ und Pressemonopolen - nicht nur in totalitären, sondern auch in freiheitlich-pluralistischen Systemen warnt. ${ }^{27}$

\subsection{Katholische Presse: „Die Fackel der Wabrbeit bochbalten"}

Die vorausgehend skizzierten Erwartungen stellt Johannes Paul II. erst recht an die 'katholische' Presse, d. h. an die Kirchenpresse und die dort tätigen Journalisten. In besonderer Weise sieht er den Beruf des katholischen Journalisten als „Sendung" zur Information und öffentlichen Meinungsbildung: „Mit der Macht der ihnen zur Verfügung stehenden Mittel und indem sie sich einer einfachen und klaren Sprache bedienen, die von den glänzenden Gaben des einzelnen Schreibers geprägt ist, mögen sie sich die erhebende Botschaft von der persönlichen und gesellschaftlichen Versöhnung zu eigen machen, zu der ich 
die Söhne und Töchter der Kirche und alle Menschen, die der Frohbotschaft des Evangeliums wohlgesonnen sind, aufgerufen habe. ${ }^{\text {“28 }}$

In seinen Ansprachen an katholische Journalisten wird deutlich, daß Johannes Paul II. hier sein Bild von dieser speziellen Presse unmittelbar aus seinem 'kommunikativen' Verständnis der göttlichen Trinität und des Offenbarungsgeschehens ableitet. „Diese 'Kundgebung' offenbart Gott im unauslotbaren Geheimnis seines einen und dreifaltigen Seins, das von 'unzulänglichem Licht' umgeben ist. Doch erkennen wir Gott durch die 'Kundmachung' Christi vor allem in seiner liebenden Zuwendung zum Menschen, in seiner 'Menschenfreundlichkeit'. "29 Die Menschwerdung Gottes deutet der Papst auch als Kommunikation zwischen Gott und Mensch. Der in Jesus Christus inkarnierte Gott ist „Meister der Kommunikation“, der zu den Menschen „aus dem Herzen, ganz in ihrer Mitte stehend" sprach. „Tatsächlich ist Kommunikation mehr als nur Äußerung von Gedanken oder Ausdruck von Gefühlen; im tiefsten ist sie Mitteilung seiner selbst in Liebe. Die Kommunikation Christi ist Geist und Leben. ${ }^{\text {30 }}$

Abgesehen von den Ansätzen einer „Theologie der Kommunikation“, die Johannes Paul II. in derartigen Äußerungen - auch mit Hinweisen und Zitaten aus "Communio et Progressio" - skizziert, wird dabei erneut die anthropozentrische Akzentuierung erkennbar. Von daher begründet er auch die Notwendigkeit für die Kirche, Kommunikation und Verkündigung mittels eigener, spezifisch katholischer Medien zu unternehmen. „Man hat gesagt, daß Zeitungskolumnen, Rundfunkmikrophone und Fernsehkameras eine Kanzel darstellen, von der die moderne Gesellschaft einen Großteil ihrer moralischen und geistigen Orientierung bezieht. Wenn das stimmt, ist es wichtig und wesentlich, daß die Kirche sich nicht nur an der Formulierung der öffentlichen Philosophien beteiligt, die die gemeinsamen Werte der heutigen Gesellschaft darstellen, sondern auch direkt auf dieser neuen Kanzel präsent ist: mit ihren eigenen Zeitungen und Zeitschriften, ihren eigenen Rundfunk- und Fernsehstationen und programmen, ihrer eigenen Stimme der Wahrheit und Liebe. “31 Die Kirche, so betont Johannes Paul II. immer wieder, hat einen Dienst „an den Kommunikatoren und einen Dienst der Kommunikation“. Dieser Dienst hat für ihn eine zweifache Funktion: innerkirchlich die Gemeinschaft in Christus und weltweit die Gemeinschaft der Menschen durch Mitformulieren der öffentlichen Philosophie und Eintreten für den Frieden zu fördern. Die Aufgabe der Evangelisierung durch die Medien nimmt für den Papst auch einen „hervorragenden Platz unter den wichtigsten Ämtern der Bischöfe" ein. ${ }^{32}$

\subsection{Katbolische Journalisten: „Publizistisches Apostolat“}

Mehr noch als jeder Journalist sind für Johannes Paul II. die katholischen Journalisten und die in katholischen Medien Schaffenden in besonderer Weise „Diener des Wortes“ (Lk 1,2), und die Kirche blickt „mit großer Sympathie und Freundschaft auf Ihre Arbeit als katholische Journalisten". In den vielen Ansprachen und Grußadressen des Papstes an katholische Journalisten entwickelt er regelrechte Elemente eines "publizistischen Apostolats". So bezeichnet er etwa in theologischer Überhöhung ihre Tätigkeit als „Berufung" oder „Sendung zur Information und öffentlichen Meinungsbildung". Immer wieder hebt er dabei 
die ,enge Verknüpfung zwischen Beruf und Moral“ hervor: „Ihr Beruf verlangt in der Tat vielfältige Opfer, grenzenlose Hingabe, dauernde Erfahrung, ständiges Bemühen um menschliche, intellektuelle und geistige Reifung. Er setzt seine Vertreter auch leicht Gefahren aus, von denen die schwerste noch immer darin besteht - gebe Gott, daß das nur unfreiwillig geschieht! - das heilige Antlitz der Wahrheit und das höchste Ideal des Guten zu trüben oder zu entstellen." ${ }^{33}$ Katholische Journalisten und ihre Medien sollen „Verstärker all dessen sein, was sich hier, am Sitz des Nachfolgers Petri, im Zentrum der Christenheit" zuträgt. ${ }^{34}$ Ihr Einsatz müsse vor allem zugunsten der Menschheit geschehen und vor allem dahin zielen, "der Wahrheit zu dienen, dem, was den Menschen positiv aufbaut, was ihn formt und besser macht. In dem Maß, in dem Sie dieses höchste Ziel verfolgen, versichere ich Ihnen ..., daß die Kirche an ihrer Seite steht, weil auch sie der Wahrheit und der Freiheit dient“ ${ }^{35}$ Einen besonderen Dienst haben katholische Journalisten in der Sicht Johannes Paul II. auch am Volk Gottes und an den Rezipienten zu leisten. Dank Informationsarbeit und Lebenshilfe sollen „noch mehr Gläubige mündig werden für das 'Zeitgespräch der Kirche', das unseren Glauben immer und jeweils neu zu stärken und in das Interesse der Öffentlichkeit zu rücken vermag, als Orientierung und Hilfe sowie als Einladung für Suchende". ${ }^{36}$ Folglich räumt der Papst den katholischen Journalisten eine „zentrale Stellung" ein, die „ihren tiefen Grund“ in der „Verbindung mit dem Papst“, in der „Treue zum Papst" und in dem „Bedürfnis ..., daß Euer Herz im Einklang mit dem Papst - dem 'ewigen und sichtbaren Prinzip und Fundament' - ... für die Einheit der Kirche ... schlägt“. Die "missionarischen Ziele“, denen sich der katholische Journalist verpflichtet fühlen soll, machen ihn für Johannes Paul II. zu einem „bevorzugten Werkzeug der Evangelisierung, die zugleich Verkündigung, Zeugnis und authentische Förderung des Menschen ist." Daher der Appell des Papstes: ,... entfaltet Euren täglichen Dienst nicht bloß mit der Euch eigenen Kompetenz, sondern auch mit jenem von Eifer und Verständnis getragenen apostolischen Geist, wie er von dem in der Kirche und in der Welt eingesetzten Jünger Christi verlangt wird. . ${ }^{* 37}$

Diese kleine Auswahl an Zitaten zeigt: Von den sich „katholisch" verstehenden Journalisten, gleich ob sie in säkularen oder in kircheneigenen Medien arbeiten, erwartet Johannes Paul II. einen besonderen Einsatz für eine zeitgemäße Evangelisierung. Das Berufsbild und -ethos des katholischen Journalisten verstärkt und überhöht der Papst zu einem anthropozentrisch ausgerichteten publizistischen Apostolat, das dem Menschen aufbauende Lebenshilfe und Orientierung in Glaubensfragen vermitteln soll. Im Bereich des Presse- und Kommunikationswesens sieht Johannes Paul II. auch ein besonderes Feld für die "Sendung der Laien“. Er setzt bei den katholischen Journalisten nicht nur wie bei ihren säkularen Kollegen - besondere Achtung gegenüber religiösen Themen voraus, sondern akzentuiert eine enge Bindung an die Kirche und besondere Treue zum Inhaber des Papstamtes.

\section{Der katholische Journalist - Idealbild mit Brüchen}

Der journalistische Alltag im Bereich kirchlicher Medien lehrt immer wieder, wobei die Methoden der Einflußnahme differenziert - und oft unauffällig - sind, daß in den kirchlichen Entscheidungen zu Medienfragen "Wollen, 
Sein und Sollen“ in Diskrepanz zueinander stehen. ${ }^{38}$ "Damit zusammen hängt die Empfindlichkeit für Kritik. Am liebsten sähe man die ausschließlich positive Berichterstattung. Der mit kirchlichen Themen befaßte Journalist wird oft als Verbündeter in Sachen 'public relations' gesehen. "39

Überprüft man G. Deussens These, daß sich bei Johannes Paul II. gegenüber einer vergangenen vertikal-hierarchischen eine mehr horizontale Sicht des Dienstes der sozialen Kommunikationsmittel durchgesetzt habe ${ }^{40}$, so ist festzustellen, daß in dem Bereich, in dem Kirche direkt Einfluß ausüben kann, d. h. im Bereich der eigenen katholischen Medien, die Erwartungen doch wieder stärker in Richtung „zweite Kanzel“ tendieren und das vertikal-hierarchische Element deutlicher im Vordergrund steht. Dies läßt sich auch an den Erwartungen Johannes Paul II. an die kircheneigenen Medien und an seinem Bild von den dort tätigen katholischen Journalisten ablesen. Wird - nachdem dies für die säkularen Medien nicht mehr möglich ist - nicht im Bereich der eigenen Kommunikationsmittel der Traum von der „treu ergebenen" Presse weitergeträumt?

Die katholische Presse steht für Johannes Paul II. vor allem im „Dienst des Papstes und des Heiligen Stuhls“, ihre Funktion für die jeweilige Ortskirche betont er nicht. Da diese Presse und die für sie tätigen Journalisten in besonderer Weise der Wahrheit und dem Papst verpflichtet seien, führt Johannes Paul II. aus: „Im übrigen werden Sie wohl verstehen, daß es einer zwielichtigen, gefährlichen und letzten Endes selbstmörderischen Haltung gleichkäme, sich von der Institution Kirche, von der hierarchischen Kirche zu distanzieren, selbst wenn Leser, die den kirchlichen Sinn etwas verloren haben, dazu auffordern. Das heißt natürlich nicht, sich auf die offiziellen Äußerungen der Kirche zu beschränken: Kirche, das ist auch das tägliche Leben der Christen und ihrer Vereinigungen. Aber die Einrichtungen der Kirche sind ihrerseits Schöpfer von Ereignissen mit sehr weitreichenden Auswirkungen, und die Ausrichtungen des Lehramtes, der verantwortlichen Bischöfe erlauben uns, die Treue des Erlebten zum katholischen Glauben zu überprüfen. “‘1

Kommunikationsfreiheit im innerkirchlichen Raum wird durch Johannes Paul II. überwiegend durch Negativkriterien bestimmt. Während er im allgemeinen Mediensektor einen Freiraum für die kirchlichen Medien als „Stimme der Wahrheit" einfordert, skizziert er für den kirchlichen Binnenraum doch stärker das Bild eines hierarchischen, von oben überprüften Dialogs, bei dem das Lehramt "Wahrheit", „Freiheit" und "Objektivität" definiert und dann diesbezüglich Parteilichkeit erwartet. Das 'Dialogforum' Kirchenpresse wird doch eher als '"zweite Kanzel' und Sprachrohr definiert, um die eine katholische Wahrheit und Weltanschauung im Konzert der pluralistischen Meinungen im säkularen Medienbereich zur Geltung zu bringen. Für den kircheninternen Raum wird zwar auch ein „christlicher Pluralismus“ zugestanden, dessen Grenzen aber bestimmen das römische Lehramt und die Bischöfe, die „Hirten der Herde“.

Bei einer restriktiven Interpretation diesbezüglicher Aussagen Johannes Paul II. ließe sich damit jede unliebsame Kritik - auch wenn sie aus Interesse und Liebe zur Kirche geschieht - unterbinden. Intern läßt sich aus dem Bild, das sich der Papst von den katholischen Journalisten macht und aus den Erwartungen, die er an sich richtet, eher das Schema eines vertikal-monologischen 'Dia- 
logforums' ableiten, bei dem die 'Hirten' ihre 'Herde' instruieren - und dabei kaum die Chance sehen, über ihre eigenen katholischen Medien auch etwas darüber zu erfahren, wie denn im 'Volk Gottes' gedacht wird.

So sind für Johannes Paul II. - und dies hebt er allein schon quantitativ deutlich häufiger hervor - die katholischen Journalisten und ihre Medien "Werkzeuge einer höheren Sendung der Wahrheit" und "Sprachrohr der Zuversicht des Evangeliums“. Problematisch wird dieses Idealbild vor allem da, wo Presse und Kanzel, also Journalismus und Pastoral, zu stark vermischt werden und der Sprachrohr-Funktion eine Ausschließlichkeit beigemessen wird.

Vor diesem Hintergrund tritt Johannes Paul II. für die Förderung einer „guten“, einer „katholischen Presse, die diesen Namen auch wirklich verdient“, ein, die damit ein „wichtiges Instrument zur Evangelisierung und Unterrichtung breiter ... Bevölkerungskreise" ist. ${ }^{42} \mathrm{Zu}$ Spannungen und Enttäuschungen muß dieses hohe Idealbild von der eigenen Presse und ihren Journalisten führen, nicht nur, weil es letztlich eine Überforderung ist (denn der Journalist vermag nicht - zumindest nicht mit der erhofften Wirkung -, Seelsorge zu betreiben; hier wird die 'Macht' der Medien doch überschätzt), sondern weil hinter diesen Vorstellungen das Bild einer "Gesinnungspresse“ steht, die „Erklären“, „Verteidigen“, „Durchsetzen" und „Beeinflussen“ soll, wie E. Dovifat die Hauptaufgaben der katholischen Publizistik zusammenfaßt. ${ }^{43}$ Die Zeit ist - auch im säkularen Bereich (vgl. das Schicksal so mancher Parteizeitung) - über den Typus der Gesinnungspresse, die etwa als katholische Presse nach dem Willen des Papstes ein 'Korrektur-Instrument' für die pluralistische, libertinistische und wertneutrale öffentliche Meinung sein sollte, hinweggegangen.

Die Generalisierung und Idealisierung in den Äußerungen Johannes Paul II. hat seinen Grund darin, daß es sich um 'Gelegenheits-Verlautbarungen' handelt. Das ist nicht nur negativ zu werten, räumt es doch der - allerdings aus diesem Grund auch notwendigen - Interpretation und Re-Lektüre zunächst ein breites Spektrum ein. Schwerwiegend ist jedoch, daß Johannes Paul II. selbst seine Vorstellungen über die Medien allgemein eher restriktiv auslegt, wenn es ihm um die katholische Presse geht. Hier gibt er, um im Bild der Kirche als „Mater et Magistra“ zu sprechen, der „Magistra“, also dem Dogma und der Hierarchie, eindeutig den Vorrang. Weil dies schnell sehr weit ausgedehnt wird, kommt es immer wieder zu Spannungen zwischen dem kirchlichen Lehramt und der eigenen Presse. Dieses Paradigma birgt aber für die Kirchenpresse auch die Gefahr, in der Konkurrenz zum säkularen Journalismus zu unterliegen, weil ihre Arbeit unter dem 'Druck von oben' bisweilen allzu deutlich zur unkritischen 'Hofberichterstattung' gerinnt, die die Mehrheit - auch der noch kirchentreuen - Rezipienten eher abschreckt; sinkende Auflagen der Kirchen- und Bistumspresse sind dafür wohl ein Indiz. Wo aber der 'Forumcharakter' der Kirchenpresse eingeschränkt und drängende Fragen und Probleme oft allzu schnell und kleinlich zum 'Tabu' erklärt und damit aus dem 'Zeitgespräch in der Kirche' ausgeklammert werden, da wird diese Presse schnell auch von den Rezipienten als 'zweite Kanzel' entlarvt. In der Zeitung sucht man möglichst umfassende Informationen der eigenen Meinung, weniger aber die Predigt.

Das von den Medien gezeichnete Idealbild und die oft bei Johannes Paul II. anklingende Vorstellung, sowohl die Medien selbst, als auch über diese die 
breite Masse beeinflussen und steuern zu können, überschätzen die Wirkungen der Presse. Ebenso führt es immer wieder zu Spannungen, daß konstruktive Kritik an der Kirche als einer "ecclesia semper reformanda" oft allzu schnell in den Verdacht der Zersetzung und des Defätismus geraten. Zum Problem wird ferner, daß Johannes Paul II. eine wesentliche Funktion, die der Presse im gegenwärtigen pluralistisch-demokratischen System zukommt, nämlich ihre Kontroll-Funktion, praktisch gar nicht erwähnt, zumindest nicht für den Aufgabenbereich der Kirchenpresse. Nicht minder problematisch ist, daß er Begriffe wie "Wahrheit" oder "Freiheit" meist ohne nähere Erläuterung einführt und damit nicht dem Umstand Rechnung trägt, daß die Kirche ihre zu verkündende "Wahrheit“ in einer Gesellschaft, die nicht mehr ohne weiteres 'die eine Wahrheit' unhinterfragt akzeptiert, sondern ein wissenschaftlich-hedonistisches Wahrheitsverständnis hat, argumentativ anders vortragen muß.

So wächst die Kluft und Widersprüchlichkeit im Bild Johannes Paul II. zwischen den allgemeinen Medien auf der einen und der kirchlichen Presse auf der anderen Seite. Für die säkulare Gesellschaft sind die Definition des Gemeinwohls sowie Wahrheitsfindung und Meinungsbildung ein wandelbarer und offener Prozeß des Suchens, der sich auch als Diskussionsprozeß und 'Streit der Meinungen' vollzieht. Der neuzeitliche Mensch ist weniger bereit, Vorgesetztes ohne ihm nachvollziehbare Argumente einfach zu übernehmen. Natürlich kann die Kirche von ihren eigenen Medien erwarten, daß diese im allgemeinen pluralistischen Meinungskonzert als Komplementärelement anders, vor allem auch mit größerer Sachkenntnis, über kirchliche Ereignisse informierten und dabei teilweise auch die „Sprachrohr"-Funktion erfüllten; die Frage bleibt aber, ob sich die Funktion der katholischen Presse darin erschöpft.

Von Otto B. Roegele stammt die Mahnung: „Ein Rückfall in eine rein prohibitive Pressepolitik, wie sie vor dem Konzil bestand, wäre nicht nur aus dem Grunde unerträglich, weil sie große Möglichkeiten des apostolischen Wirkens ungenutzt ließe. Wichtiger noch erscheint es mir, daß auch der Vatikan nicht einfach daran vorbeigehen kann, daß jetzt ein allgemeines Konzil die Bedeutung der Kommunikationsprozesse und der sie in Gang haltenden Menschen und Mittel in aller Form erörtert hat, daß dieses Konzil das von zwei Päpsten - Pius XII. und Johannes XXIII. - ausdrücklich erklärte Recht der Menschen auf wahrheitsgemäße Information über sie betreffende Fragen anerkannt hat und daß dadurch ein neuer Bewußtseins-, aber auch ein neuer Rechtszustand eingetreten ist, der eine neue Einstellung zu und eine neue Handhabung der Kommunikationsmittel erforderlich macht. ${ }^{\text {" }}{ }^{44}$ Es gibt leider - wie etwa auch die medienkritischen, wenn nicht gar -feindlichen Passagen in der jüngsten „Instruktion der römischen Kongregation für die Glaubenslehre über die kirchliche Berufung des Theologen “ ${ }^{45}$ belegen, deutliche Anzeichen für eine gegenteilige Tendenz.

\section{Anmerkungen:}

1 Johannes Paul II., Ansprache vor italienischen Bischöfen am 04.01.1982, zit. nach: Katholische Presse - eine Presse für den Menschen, Informationsbroschüre der Arbeitsgemeinschaft Katholische Presse (AKP), Sankt Augustin o. J.

2 Vgl. zur Literatur: Johannes Paul II., Wort und Weisung im Jahr 1978-1981, Deutsche Ausgabe: Verlag Butzon \& Bercker, Kevelar (Abk.: WuW); ders., Der Apostolische 
Stuhl - Ansprachen, Predigten und Botschaften des Papstes - Erklärungen der Kongregation. Vollständige Dokumentation, hg. v. Sekretariat der Deutschen Bischofskonferenz, Bonn/Köln 1982ff. im J. P. Bachem-Verlag (Abk.: AS; setzt die Reihe WuW fort); ders., Verlautbarungen des Apostolischen Stuhls, hg. v. Sekretariat der Deutschen Bischofskonferenz, Bonn 1975ff. (in dieser Druckschriftenreihe veröffentlicht das Sekretariat ab 1975 unter fortlaufender Nummerierung Erklärungen der Kongregation, Schreiben der Päpste und Predigten/Ansprachen Johannes Paul II. bei seinen Auslandsreisen; Abk.: Verlautbarungen/Nr. ..); ders., Welttag der sozialen Kommunikationsmittel - Botschaften und Leitworte seit Communio et Progressio (1971), Arbeitshilfen Nr. 64, hg. v. Sekretariat der Deutschen Bischofskonferenz, Bonn 07.05.1989 (Abk.: Arbeitshilfen 64/Welttag; „Inter mirifica“, Dekret über die sozialen Kommunikationsmittel, in: K. Rahner/H. Vorgrimler (Hg.), Kleines Konzilskompendium, Freiburg 41980, 91-104 (Abk.: IM); "Communio et Progressio" - Pastoralinstruktion der Päpstlichen Kommission für die Instrumente der sozialen Kommunikation, Lateinisch-deutsch, kommentiert von H. Wagner, Trier 1971 (Abk. Cep).

3 Vgl. A.-F. Utz/J.-F. Groner, Aufbau und Entfaltung des gesellschaftlichen Lebens. Soziale Summe Pius' XII., 3 Bände, Freiburg 1954ff.; W. Hamerski, Reden Papst Pius XII. zu Fragen der Publizistik, in: Publizistik 8 (1964), 611-631.

$4 \mathrm{Utz} /$ Groner, a. a. O., Nr. 2133.

5 Vgl. G. Deussen, Ethik der Massenkommunikation bei Papst Paul VI., München/ Paderbom/Wien 1973.

6 So fragt G. Deussen, „Anthropologische Wende“ in der kirchlichen Soziallehre zur Massenkommunikation - Der Beitrag Papst Johannes Paul II., in: CS 2/1985, 94-114, 95.

7 Ebd

8 E. Bordfeld, Johannes Paul II. - Ein Papst für die Medien, Medien für den Papst, in: CS 2/1983, 103-114, 103.

9 M. Schmolke, Zehn Jahre nach „Communio et Progressio“, in: CS 4/1981, 283-289, 284 .

10 Johannes Paul II., Botschaft zum 18. Welttag der sozialen Kommunikationsmittel 1984, in: AS 1984, 1246 oder in: Arbeitshilfen 64/Welttag, 154-158, 156.

11 Gregor XVI., Enzyklika „Mirari vos", in: Acta Gregorii XVI., Ausgabe A. Bernasconi, Rom 1901, 169-174; in der er die "verderbliche Pressefreiheit" und andere "moderne Freiheiten" geißelt; vgl. J. Gelmi, Das Papsttum bis zum Ersten Weltkrieg, in: B. Moser (Hg.), Das Papsttum, München 1983, 248.

12 Johannes Paul II., Ansprache an Journalisten in Krakau am Ü10.06.1979, in: Verlautbarungen 10/1979, 115; vgl. auch Ansprache an Journalisten in Mexiko-City am 31.01.1979, in: Verlautbarungen 5/1979, 104f.; Ansprache an Journalisten auf dem Rückflug von der Afrikareise am 12.05.1970, in: Verlautbarungen 18/1980, $127 \mathrm{f}$. 13 IM Nr. 3.

14 CeP Nr. 126-128.

15 Johannes Paul II., Botschaft zum 18. Welttag der sozialen Kommunikationsmittel 1984, in: AS 1984, 1247; auch in: Arbeitshilfen 64/ Welttag, 156f.

16 Vgl. dazu die erste Enzyklika Johannes Paul II. „Redemptor hominis“ (1979), Deutsche Ausgabe: Verlag Butzon \& Bercker, Kevelaer/Verlag Johann M. Neumann, Würzburg 1979. Dort heißt es u. a. in Kapitel 14: „Dieser Mensch ist der erste Weg, den die Kirche bei der Erfüllung ihres Auftrages gehen muß; ein Weg, der von Christus selbst vorgezeichnet ist und unabänderlich durch das Geheimnis der Mensch werdung Gottes und der Erlösung führt." Diesen Satz zitiert Johannes Paul II. in seiner Ansprache an Repräsentanten von Wissenschaft und Kunst in Wien am 12.09.1983, in: AS 1983, 625.

17 Vgl. Johannes Paul II., Ansprache an Vertreter von Wissenschaft und Kultur am 25.02.1981 in Hiroshima/Japan, in: WuW 1981, 330-337; ders., Ansprache an Repräsentanten von Wissenschaft und Kultur in der Soyang-Universität Seoul/Südkorea am 05.05.1984, in: AS 1984, 327-332; ders., Botschaft zum 15. Welttag der sozialen Kommunikationsmittel 1981, in: WuW 1981, 474-479 oder in: Arbeitshilfen 64/Welttag, 121-126. 
18 G. Deussen, „Anthropologische Wende“, in: CS 2/1985, 94-114.

19 IM Nr. 11.

20 Johannes Paul II., Ansprache an Künstler und Publizisten in München am 18.11.1980, in: Verlautbarungen 25/1980, 190.

21 Ders., Ansprache an Journalisten in Mexiko-City, a. a. O., 105; ders., vgl. Ansprache an Journalisten beim Rückflug von der Afrikareise, a. a. O., 171f.; ders., Ansprache an Journalisten in Manila/ Philippinen am 21.02.1981, in: WuW 1981, $218 \mathrm{f}$.

22 Ders., Ansprache an Journalisten in Mexiko-City, a. a. O.; ders., Ansprache an Journalisten in Rom am 27.01.1984, in: AS 1984, 920-927.

23 Ders., Ansprache an Journalisten beim Rückflug von Afrika, a. a. O., 171.

24 Ders., Ansprache an Repräsentanten der Medien in Madrid am 02.11.1982, in: AS $1982,709$.

25 Ebd.

26 Ders., Ansprache an die beim Hl. Stuhl akkreditierten Journalisten am 22.05.1982, in: AS 1982, 1117-1120, 1119.

27 Ders., ebd., 1118f.; ders., Botschaft zum 15. Welttag der sozialen Kommunikationsmittel 1981, in: Arbeitshilfen 64/Welttag, 121-126.

28 Ders., Ansprache an Journalisten des katholischen Presseverbandes Italiens am 14.02.1983, in: AS 1983, 788-792, 789.

29 Ders., Enzyklika „Dives in misericordia“ (1980), Deutsche Ausgabe, hg. vom Sekretariat der Deutschen Bischofskonferenz, Bonn 1980, 5.

30 CeP Nr. 11.

31 Johannes Paul II., Ansprache an die Vollversammlung der Päpstlichen Kommission für die sozialen Kommunikationsmittel am 27.02.1986, in: CS 2/1986, 166-169, 168; vgl. ders., Schlußdokument der Außerordentlichen Bischofssysnode 1985, in: AS 1985, 1866-1885, 1866.

32 Ders., Ansprachen u. a. an Bischöfe Australiens am 02.07.1983, in: AS 1983, 12911295; ders., Ansprache an deutsche Bischöfe am 21.01.1983, in: AS 1983, 1310-1315; ders., Ansprache an US-amerikanische Bischöfe am 22.10.1983, in: AS 1983, 1469f., und am 09.09.1983, in: AS 1983, 1452-1456.

33 Ders., Ansprache an Journalisten des katholischen Presseverbandes Italiens, a. a. O., 789.

34 Ebd.

35 Ebd.

36 Ders., Ansprache an Teilnehmer des Romseminars der Arbeitsgemeinschaft Katholische Presse (AKP) am 06.04.1984, in: AS 1984, 1138 f.

37 Ders., Ansprache zum 50jährigen Jubiläum von Radio Vatikan am 12.02.1981, in: WuW 1981, 172-175, 173-175.

38 K. Kraemer, Kirche und Medien - ein gestörtes Verhältnis, in: Die Zeitung als Persönlichkeit - Festschrift für Karl Bringmann, Düsseldorf 1982, 147.

39 H. S. Ruppert, Kirchliche Ereignisse mediengerecht präsentiert, in: CS 3/1982, 204$210,207$.

40 G. Deussen, „Anthropologische Wende“ (s. Anm. 6).

41 Johannes Paul II., Ansprache an Redaktionsmitglieder und Leser der französischen katholischen Tageszeitung „La Croix“ am 23.04.1983, in: AS 1983, 903-913, 912.

42 Ders., Rundfunkbotschaft beim Besuch der katholischen Rundfunkstation in Quito/ Ecuador am 30.01.1985, in: Verlautbarungen 61/1985, 68-71.

43 E. Dovifat, Handbuch für Publizistik, Bd. I, Berlin 1968, 5.

44 O. B. Roegele, Kirche und Kommunikationsmittel in und nach dem Konzil, in: „lebendige Kirche“, Mitteilungsblatt des Diözesankomitees der Katholikenausschüsse in der Erzdiözese Köln, Rom-Sonderheft 1965/66, 6-14.

45 Instruktion der römischen Kongregation für die Glaubenslehre über die kirchliche Berufung des Theologen vom 24.05.1990; Deutsche Ausgabe hg. vom Sekretariat der Deutschen Bischofskonferenz, Bonn 1990, vor allem 17ff. 


\section{SUMMARY}

Completely in the tradition of Vatican II. and its decree "Inter Mirifica" as well as of the Pastoral Instruction "Communio et Progressio", John Paul II. points out the necessity for the Church to use the general mass media, but also - if possible - to dispose of her own means of communication. He regards the media, and above all the Catholic media, as an appropriate "instrument" to evangelize the world. He strongly emphasizes the idea that the Catholic press should obtain a certain margin within the sphere of general media in order to give effect to the position of the Church there, finally speaking as "one voice" in view of the pluralism of opinions. Unlike any of his predecessors, Pope John Paul II. seeks contact with the media and calls the journalists his "friends". At the same time, however, he analyzes very critically the "power of the media", be it positive or negative. He considers the concepts of "truth" and of "freedom" to be the primary criteria for the work of journalists; he usually does not define these concepts, but he regards them as generally accepted. By qualifying the work of journalists - especially the of Catholic journalists - as "mission", "vocation", "service to the truth", "witness" or even "apostolate" the Pope uses terms which are not appropriate in this context from the theological point of view. Moreover he unterlines that Catholic journalists have to be closely connected with the Hierarchy, the Magisterium and above all with the Pope. John Paul II. defines the freedom of communication within the Church particularly by negative criteria. He insists on the idea that the Catholic Press - complementary or even contrary to the secular press has to fulfill the function of an apologetic "second pulpit" which affirms the Church and the faith. Also for the benefit of the Church, he does not appreciate criticism very much. Consequently, according to John Paul II., the Catholic journalist is rather an "instrument" and a "spakesman" of the Magisterium and the Pope.

\section{RÉSUMÉ}

C'est parfaitement dans la tradition du Concile Vatican II et de son décret "Inter mirifica" ainsi que de l'Instruction pastorale "Communio et progressio" que Jean-Paul II. souligne la nécessite pour L'Eglise d'utiliser les mass médias géneraux, mais de disposer également, si possible, de ses propres moyens de communication. Les médias, surtout les médias catholiques, lui paraissent un "instrument" approprieé à l'évangélisation du monde. Il tient à souligner l'idée qu'il faut accorder à la presse catholique un espace libre dans le domaine des médias généraux pur qu'elle y mette en relief la position de l'Eglise, représentant en fin de compte une seule voix face au pluralisme des opinions. Comme aucun de ses prédécesseurs le Pape Jean-Paul II. cherche le contact avec les médias et dit que les journalistes sont ses "amis"; toutefois il analyse d'une facon très critique le "pouvoir des médias", qu'il soit positif ou négatif. Il considère les notions de "vérité" et de "liberté" comme les critères primordiaux pour le travail du journaliste; en général il ne dèfinit pas ces notions mais il les tient pur généralement acceptées. En qualifiant le travail des journalistes - et surtout celui des journalistes catholiques - de "mission", "vovation", "service à la vérité", "témoignage" ou même "apostolat" le Pape utilise dans ce contexte des notions non appropriées du point de vue théologique. De même il souligne le lien étroit qui doit exister entre les journalistes catholiques et la Hiérarchie, le Magistère et spécialement le Pape. Jean-Paul II. définit la liberté de communication dans le domaine de l'Eglise sourtout par des critères négatifs. Il insiste sur l'idée que la presse catholique en complément ou même contraire à la presse séculière - doit exercer la fonction "d'une deuxième chaire" apologétique affirmant l'Eglise et la foi. Egalement en vue du bien de l'Eglise il n'apprécie pas tellement de la critique. Donc selon Jean-Paul II. le journaliste catholique est plutôt un "instrument" de l'évangélisation et un "porte-parole" du Magistère et du Pape.

\section{RESUMEN}

Siguiendo la tradición del II Concilio Vaticano y de su decreto "Inter mirifica" así como de la Instrución pastoral "Communio et progressio", el Papa Juan Pablo II. destaca la necesidad para la Iglesia de utilizar los medios de comunicación de masas generales, pero de disponer también, si es posible, de propios medios de comunicación. Los me- 
dios, sobre todo los medios católicos, le parecen un "instrumento" apropiado para la evangelización del mundo. Subraya especialmente la idea de que hay que dar a la prensa católica un espacio libre en el campo general de los medios de comunicación para que ponga de relieve allí la posición de la Iglesia representando, al fin y al cabo, una "sola voz" ante el pluralismo de opiniones. Como ninguno de sus antecesores, el Papa Juan Pablo II. busca el contacto con los medios; dice que los periodistas son sus "amigos", pero al mismo tiempo analiza de una manera muy crítica el "poder de los medios" que sea positivo o negativo. Considera los términos "verdad" y "libertad" como los criterios primordiales para el trabajo del periodista. Normalmente no define estos términos sino los da por generalmente aceptados. Calificando el trabajo de los periodistas - sobre todo de los periodistas católicos - de "misión", "vocación", "servicio a la verdad", "testimonio" o incluso "apostolado" el Papa utiliza términos teológicos en este contexto non apropiados. Igualmente destaca la vinculación necesariamente estrecha de los periodistas católicos a la jerarquia de la Iglesia, al Magisterio y, especialmente, al Papa. Juan Pablo II. define la libertad de comunicación en el ambito de la Iglesia sobre todo por criterios negativos. Sin embargo, insiste en la idea de que la prensa católica - como complemento o incluso en oposición a la prensa secular - tiene que cumplir la función de una "segunda catedra sagrada" apologética que afirma la Iglesia y la fe. Igualmente en vista del bien de la Iglesia no aprecia comentarios críticos. Por lo tanto, según Juan Pablo II., el periodista es mas bien un "instrumento" de la evangelización y un "portavoz" del Magisterio y del Papa. 